\title{
Bemerkungen zur Bewertung der Montagegerechtheit bei der Planung verfahrenstechnischer Anlagen
}

\author{
Eberhard Wegener
}

Ein technisches Gebilde wird eindeutig und vollständig durch seine geometrischen, stofflichen und Zustandseigenschaften beschrieben. Eine verfahrenstechnische Anlage entsteht planerisch als Stufenprozess, der Planer ist gezwungen, auf der jeweiligen Konkretisierungsstufe eine Beurteilung seiner Gestaltungslösung vorzunehmen und Entscheidungen zu treffen, teilweise auf niedrigem Konkretisierungsniveau, d. h. sie tragen oft nur vorläufigen Charakter und müssen in den folgenden Planungsstufen angepasst werden, so auch die montagegerechte Gestaltung von Teillösungen.

\section{Problemsituation}

Wenngleich Klarheit darüber herrscht, dass in den einzelnen Konkretisierungsstufen auch über die montagegerechte Gestaltung von Teillösungen geurteilt werden muss, so ist zu prüfen, was und wie beurteilt werden kann. Die besonderen Probleme der Gestaltung verfahrenstechnischer Anlagen komplizieren den Beurteilungsprozess, da

- durch die Anlage als Unikat jedesmal neue Bedingungen für die Montage und damit unterschiedliche Zielkriterien entstehen (z. B. Neubau-Erweiterung bei laufendem Betrieb),

- sich aufgrund der unterschiedlichen Zielkriterien Probleme bei der Auswahl der Bewertungskriterien ergeben, weil wissenschaftliche Ergebnisse zur Bestimmung einer ausreichenden Zahl von Bewertungskriterien (allgemeine Formulierungen spezifischer Merkmale) und Kennzahlen (quantitativer Ausdruck der Bewertungskriterien) bisher nicht vorliegen. Es ist daher zu entscheiden, welche und wieviele Bewertungskriterien in der jeweiligen Prozessstufe notwendig sind, um mit ausreichender Genauigkeit eine Gestaltungslösung montagetechnologisch begründen zu können. Gleichzeitig sind Überlegungen für eine Rangfolgebestimmung und eine Gewichtung notwendig, da allein die Unterschiedlichkeit im Gebrauch oder Weglassen von Bewertungskriterien für das Bewerten der gleichen Variante zu differenzierten bzw. sogar gegensätzlichen Ergebnissen fuihrt,

- infolge des Stufenprozesses bei der Entwicklung der Gestaltungslösung die Bewertungskriterien wechseln; als Konsequenz muss die Festlegung der veränderten Rangfolge die Auswirkungen in vor- und nachgelagerten Planungsstufen erfassen,

- praktische Variantenvergleiche stets die Analyse der konkreten Situation verlangen. Im allgemeinen liegen aber nicht für jede ausgearbeitete Gestal- tungsaufgabe Varianten aus montagetechnologischer Sicht vor, denn Ausrüstungsanordnungen und Führung der Rohrleitungen werden bereits z. B. nach distanzminimierenden Prämissen vorgegeben und die gestaltungsbezogene Bewertung muss komplexen Charakter tragen, d. h. sie muss uiber die rein montagetechnologische Betrachtung hinausgehen und andere Gewerke miteinbeziehen. Es ist daher nicht möglich, alle qualitativen Faktoren auf einen quantitativen Nenner zu bringen.

Da die montagegerechte Gestaltung nur eine Teilqualität des Erzeugnisses Anlage und die Wertigkeit der Montagegerechtheit innerhalb der Entwicklung der Gesamtlösung noch ungeklärt ist, führen Vorschläge wie von Plankenbichler et al. [1] nicht zum Erfolg, da die Bewertung der Qualität die Quantifizierung ihrer Qualitätsseigenschaften voraussetzt. Auch Pappritz [2] sieht die Schwierigkeiten vor allem darin, ,dass die der unmittelbaren und konkreten Wertbestimmung notwendigerweise vorgelagerten Fragen auf recht unterschiedlichen Ebenen liegen und in verschiedenen Abstraktionsrichtungen des Denkens variieren“. Im Ergebnis der Diskussion zur Bewertung der Montagegerechtheit muss festgestellt werden, dass diese nicht durch eine explizierte Kennzahl darstellbar ist. Damit verbleibt für die als notwendig erkannte Beurteilung nur die Anwendung eines Schätzverfahrens.

\section{Beurteilung der Gestaltungslösung im Dialog}

Wird als Schätzung die individuelle oder im Team aufgrund von Erfahrungen getroffene Angabe der wahrscheinlichen Merkmale vorhandener oder objektiv möglicher Sachverhalte angesehen, stellen Kenntnisse des Planers über montagetechnologische Belange und über die möglichen Maßnahmen zu ihrer Berüicksichtigung eine Voraussetzung dar. Aufgrund der Planungsstufen gestattet das Schätzverfahren die kontinuierliche Präzisierung der Beurteilungen durch Weiterentwicklung der Beurteilungszustände, der -objekte und damit auch der -maßstäbe, die Sicherheit der Beurteilung nimmt zu mit der Vervollständigung der Planungsunterlagen und damit steigendem Informationsgehalt. Da nicht prinzipiell nach jeder Teilgestaltung eine Beurteilung größeren Umfanges notwendig ist, werden 3 Stufen der Beurteilung bei der Anlagengestaltung auf der Grundlage argumentativer oder qualitativer Methoden vorgeschlagen, die jeweils mögliche und notwendige Sachverhalte beinhalten. 


\subsection{Beurteilung in der ersten Stufe - Gestaltungsentwurf}

Als erforderliche Unterlagen dienen der Lageplan, eine Objektuibersicht, der Grobaufstellungsplan, Grobtrassenpläne, die Grob-Baustruktur und erste Angaben über Masse/Abmessungen der Hauptausrüstungen. In Form von Konsultationen erfolgt eine Beurteilung montagetechnologischen Belange in folgendem Umfang:

\section{Montageeinheit:}

- Überprüfung der Transportfähigkeit und der maximalen Begrenzungen (Masse, Abmessungen, Transportmittel, Transportweggeometrie) zur und auf der Baustelle,

- Überprïfen auf Übereinstimmung mit der Hubleistung der vorhandenen Hebezeuge fuir Entladen und Montage,

- Überpuifen auf evtl. Beschränkungen in Masse/Geometrie aufgrund von Transportbedingungen, montagetechnologischen oder baulichen Randbedingungen sowie möglicher Vormontagen auf der Baustelle oder notwendigen Kontrollmontagen beim Hersteller,

- Überprïfen auf bereits erkennbare Forderungen an die bauliche Gestaltung (Montageöffnungen, Erdstabilisierungsmaßnahmen) oder an den Einsatz spezieller Technologien bzw. ausrüstungsspezifischer Montagehilfsmittel,

- gegebenenfalls bereits Anforderungen an die Gestaltung der Montageeinheiten aus dem Lastfall Montage.

\section{Anlagengestaltung - Ausrüstungsanordnung:}

- Überprüfung der Zugänglichkeit zum Baufeld/Einbauort fuir die Montageeinheit, Transport- und Hebezeuge, Einschätzung des notwendigen Platzbedarfes für Entladung, Zwischenlagerung, Vormontagen, der Abmessungen der Versorgungskorridore zwischen den Teilanlagen,

- Überprïung der Führung von Rohrbrücken, Trassen und kanalverlegten Rohrleitungen unter Berücksichtigung des Realisierungsablaufes und der Zugänglichkeit für Hebezeuge zum Baufeld,

- Überprüfung der Stellung der Montageeinheit im Produktionsprozess und der bei Ausfall zu berücksichtigenden Faktoren hinsichtlich des vorgesehenen Standortes, für Demontage udgl.,

- Einschätzung der Lage/Größe der Zentralen Baustelleneinrichtung,

- Überprifung der Einbauorte der Montageeinheiten, notwendiger Hebezeuge, deren Standort, Flächenbedarf für Zufahrt, Auf-, Ab-, Umrüsten, ausreichende Befestigung, Ermittlung zu erwartender Beschränkungen, Art der Aufstellung,

- Ermittlung möglicher Montagehöhen, Sicherung der montagetechnologisch erforderlichen Mindestabstände um die Montageeinheiten,

- Überprüfung möglicher Montagetechnologien, Ermittlung montagetechnologischer Besonderheiten, technologisch oder bauablaufbedingter Montagereihenfolgen, Aussagen zum Einsatz spezifischer Montagehilfsmittel, Ermittlung von Abhängikeiten von Bau/Stahlbau,

- Ermittlung spezieller Anforderungen an die Gestaltung, die Masse/Geometrie der Montageeinheiten, die Zufahrtswege, die Baustelleneinrichtung, die bauliche Gestaltung und die Montageorganisation,
- Einschätzung der zu erwartenden Beeinträchtigungen oder Behinderungen durch Einengung des Baufeldes, benachbarte Anlagen, Rohrbrücken, wachsende Bebauung des Baufeldes, Ex-Schutz der Hebezeuge,

- Ermittlung von Zusatzbeanspruchungen (Lastfall Montage) fuir den Baukörper und die Montageeinheiten.

\section{Bauliche Gestaltung:}

- Einschätzung von Problemen, die sich für die Montage aus der Gliederung der Anlage und der Bauweise ergeben, Möglichkeiten des Einschwenkens von Ausruistungen in den Baukörper,

- Ermittlung spezieller Anforderungen an die Gestaltung von Montageeinheiten aus Zufuihren und Auflagern sowie baulichen Begrenzungen für die Demontage, notwendige stationäre Arbeitsmittel und Hilfsvorrichtungen als Montagehilfen,

- Überprïfen der Zugänglichkeit zum Einbauort, Montageöffnungen, Belastbarkeiten, Flächenangebote für Montagevorrichtungen und -operationen, Vertikalund Horizontal-Transportmöglichkeiten,

- Überprüung der zusätzlichen Belastungen aus Transportlasten, Montagestabilisisierung, Krafteinleitungspunkten in den Baukörper.

\section{Kopplungen (Rohrleitungen):}

- Einschätzung der sich aus der Verlegungsart zwischen den Teilanlagen ergebenden Probleme, Verflechtungsgrad, Möglichkeiten der Vorfertigung und Vormontage von Rohrleitungen, mögliche Probleme bei Rohrleitungen großer Nennweite oder bei speziellen Werkstoffen, Zugänglichkeit für Hebezeuge,

- Ermittlung montagetechnologisch oder ablaufbedingter Montagereihenfolgen und Ableiten von Gestaltungsforderungen,

- Ermittlung möglicher Behinderungen/Beeinträchtigungen für die Rohrleitungsmontage.

Die Überprüfung der grundlegenden beeinflussenden Faktoren, ihrer Auswirkungen usw. ist prinzipiell durch Check-Listen auf dieser Basis möglich, da sie jedoch nur den jeweiligen Stand der Fertigungs-, Transport- oder Montagetechnologie (-möglichkeiten) wiedergeben und die Dialogfuihrung zu diesem Problem nicht ausschließen, ist einer argumentativen Beurteilung der Vorzug zu geben.

\subsection{Beurteilung in der zweiten Stufe - Gestaltungslösung}

Die Beurteilung erfolgt mit dem anhand der Erkenntnisse aus der ersten Beurteilungsstufe überarbeiteten Aufstellungsplan der Ausrüstungen, von Rohrleitungs- und Trassenplänen, ilsometrischen Rohrleitungszeichnungen, von Apparateskizzen und Anfragezeichnungen sowie Fundament- und Durchbruchplänen, die eine Einschätzung der Detailprobleme erlauben. Beurteilungsschwerpunkte sind nun:

\section{Montageeinheit:}

- Bestimmung des optimalen Anlieferungszustandes, der Möglichkeit der weiteren Vormontage bzw. Komplettierung auf der Baustelle, 
- Überprüfung der Montagefähigkeit, der erforderlichen Anschlagpunkte für das Zufuihren, das Herstellen der Einbaulage und der Lagesicherung in Anzahl und Anordnung,

- Überprüfung der vorgesehenen Maßnahmen zur Sicherung der Lagerfähigkeit und Qualitätserhaltung,

- Überprüfung auf zusätzliche Montagebeanspruchungen durch Auflagern von Montagehilfen, Begehung oder Rüsthilfen.

\section{Anlagengestaltung - Ausrüstungsanordnung:}

- Überprüfung des Flächen- und Raumbedarfes/-angebotes fuir die Baustelleneinrichtung, der Aufstellund Rüstflächen fuir die Hebezeuge, der Vormontageund Montageflächen einschl. der Transportwege,

- Ermittlung zusätzlicher Anforderungen für Montagehilfsmittel sowie baulicher Angaben,

- Überprüfen auf Vermeidung unnötiger Einrüstungen und Ermittlung von Anforderungen an die Gestaltung von Montageeinheiten hinsichtlich der konstruktiven Berüicksichtigung von Rüsthilfen.

\section{Bauliche Gestaltung:}

- Überprïfung des Flächen- und Raumbedarfes/-angebotes für Montageflächen und -wege, der Belastbarkeiten,

- Überprüfung auf Vorhandensein notwendiger stationärer Arbeitsmittel, Montagehilfen, Lasteintragstellen,

- Überprüfung der notwendigen Einrichtungen für die Aufnahme der Auflagerung von Ausruistungen und Rohrleitungen sowie deren maßliche Genauigkeitsvorgabe in Abmessungen, Lage und Anordnung.

\section{Kopplungen (Rohrleitungen):}

- Einschätzung des möglichen Vorfertigungsgrades unter Beruicksichtigung des Montagefortschritts und der Verlegekriterien sowie zweckmäßige Lage der Fügestellen,

- Überprüfen des Raum- und Flächenangebotes zur Rohrleitungsmontage, der zweckmäßigen Verbindung zwischen den Ausrüstungen, Unterstuitzungen und Halterungen,

- Überprifung auf zu erwartende Passungsprobleme, Passendenfestlegung,

- Überprüfen der montagetechnologisch erforderlichen Abstände zwischen den Rohrleitungen sowie zu Ausrüstungen und baulichen Einrichtungen,

- Überprüfen der Armaturenanordnung auf Montagefähigkeit (Demontage, stationäre Montagehilfen),

- Überprüifung möglicher Beeinträchtigungen/Behinderungen durch den laufenden Betrieb, die bauliche Umgebung, Sicherheitsforderungen, Ex-Schutz.

\subsection{Beurteilung in der dritten Stufe - Vor- und Nachbereitungsmöglichkeiten}

Sind die Detailfragen aus der 2. Beurteilungsstufe geklärt und die notwendigen Anpassungen erfolgt, können auf der Grundlage der konkreten Planungsunterlagen für die einzelnen Teillösungen die Möglichkeiten einer rationellen Vor- bzw. Nachbereitung gepruft werden. Ihr Einfluss auf den Montageaufwand und vor allem auf die Montagezeit wird häufig unterschätzt. Dabei geht es um eine effektive Vor- bzw. Nachbereitung der Montageeinheiten durch

- Überprifung auf Festlegungen zu qualitätserhaltenden Maßnahmen bis zu Montage in den Planungs-/ Konstruktionsunterlagen,

- die Prüfung auf die Möglichkeit, die vorgesehenen Prüfund Kontrollprozesse rationell durchfuihren zu können,

- die Überpruifung der rationellen Durchfuihrbarkeit von Leistungen anderer Gewerke.

\section{Anwendbarkeit der Beurteilungsmethode}

Die Aufstellung eines allgemeinen Zielrahmens scheitert an der Festlegung notwendiger Bewertungskriterien und deren Gewichtung. Wenngleich unterschiedliche Auffassungen zur notwendigen Anzahl der Bewertungskriterien und deren Rangfolgebestimmung bestehen (sie schwanken zwischen 3 und 20, Gäfgen [3] begründet 6 als sinnvoll) und die Sinnfälligkeit einer Gewichtung (Lowka [4] bereits 1975), so zeigt Abbildung 1 auch die Möglichkeiten/Grenzen der Einflussnahme der Montage auf den Gestaltungsprozess). Auch bei der Auswahl der Beurteilungskriterien, die letztlich zu den Überprüfungsmaßnahmen in den vorgeschlagenen drei Beurteilungsstufen fuihren (Abbildung 2), tritt die Problematik auf, dass Beurteilungsergebnisse in den einzelnen Beurteilungsbereichen zum Erkennen von Folgeproblemen führen, für deren Beurteilung wieder sog. Ersatzkriterien formuliert werden müssen.

Die vorgeschlagene Schätzmethode stellt offensichtlich die effektivste Möglichkeit dar, Gestaltungslösungen unter dem Gesichtspunkt der Montage zu beurteilen. Inwieweit das Beurteilungsprogramm vollständig im Dialog abgearbeitet werden muss, hängt weitgehend von der methodischen Vorgehensweise und vom subjektiven Erfahrungsschatz des Planers ab. Es garantiert jedoch in jedem Fall die Berüicksichtigung aller zu erwartender Montageprobleme.

\section{Ausblick}

Der Zwang nach aufwandsarmen Anlagen für die Montage erfordert eine zielgerichtete und effektive Schaffung montagegerechter Lösungen. Die Schwierigkeiten liegen in der nachgewiesenen Vielfalt der zu berüicksichtigenden Einflussfaktoren. Es wird als empfehlenswert angesehen, als methodisches Hilfsmittel ein Regelwerk auszuarbeiten, das einerseits den Einsatz unmittelbar in der praktischen Arbeit des Planers findet und sowohl als Arbeitsmittel zur bewussten montagegerechten Gestaltung als auch zur Überprüfung von Gestaltungslösungen dienen kann, andererseits seine Verwendung in der Ausbildung zur Befähigung zur methodischen Arbeit, auch unter Beachtung des Stoff-Zeit-Problems in der Ingenieurausbildung. Es wäre jedoch ein Irrtum zu glauben, dass sich mit einem Regelwerk allein eine effektive Montagevorbereitung und -durchfuihrung ergibt, es hängt von der Motivation der Beteiligten ab, inwieweit vorteilhafte Arbeitsmethoden angewendet werden. Die vorgeschlagene Vorgehensweise kann hierzu einen Beitrag leisten. 


\section{Literaturverzeichnis}

[1] Plankenbichler, Krug, Handschur: Die Bewertung einer komplizierten Anlage und Schlussfolgerungen für spezielle Methodiken der Qualitätsbewertung. Standardisierung und Qualität 20 (1974) 7, S. 281-285

[2] Pappritz, P.: Das Wertproblem im technischen Schaffen Fragen zur Diskussion, Positionsbestimmung, Anregung zum weiteren Dialog. Wiss. Z. Techn. Univ. Dresden 31 (1982) 5, S. 55-61

[3] Gäfgen, G.: Theorie der wirtschaftlichen Entscheidungen. Mohr Verlag Tübingen 1968

[4] Lowka, D.: Gewichtungen bei Bewertungen. Konstruktion 27 (1975) 9, S. 345-347

\section{Autor}

Prof. Dr.-Ing. Eberhard Wegener

Technische Fachhochschule Wildau Fachbereich Ingenieurwesen/Wirtschaftsingenieurwesen Tel. (0 33 75) 508-169

E-Mail: wegener@vt.tfh-wildau.de

\begin{tabular}{|c|c|c|c|c|c|c|}
\hline $\begin{array}{l}\text { Elnilusse aut } \\
\text { Kriterien } \\
\text { Gestaltungs- } \\
\text { objekt }\end{array}$ & $\begin{array}{l}\text { Charakte } \\
\text { Haupt- } \\
\text { merkmal }\end{array}$ & $\begin{array}{l}\text { eristisches } \\
\text { Zusatz- } \\
\text { merkmal }\end{array}$ & $\begin{array}{l}\text { Qualitativer } \\
\text { Einfluß auf } \\
\text { Montage- } \\
\text { aufwand }\end{array}$ & $\begin{array}{l}\text { Beeinflußbar } \\
\text { aus Sicht der } \\
\text { Montage } \\
\text { (Merkmal) }\end{array}$ & $\begin{array}{l}\text { Beurteilungskriterien für die } \\
\text { Montagegerechtheit } \\
\text { Z.B. }\end{array}$ & Bemerkungen \\
\hline \multirow[t]{10}{*}{ Anlage } & Standort & $\begin{array}{l}\text { Neubau } \\
\text { Rekonstr. }\end{array}$ & $\begin{array}{l}\text { groß } \\
\text { sehr gro } B\end{array}$ & kaum & $\begin{array}{l}\text { Zugänglichkeit für Montageeinheiten, } \\
\text { Arbeitsmittlel, } \\
\text { Behinderungen/Erschwernisse }\end{array}$ & $\begin{array}{l}\text { Sowohl vom Betrieb zur Baustelle, als auch } \\
\text { Zentrale Baustelleneinrichtung/Vormontageplatz } \\
\text { zum Einbauort, Mehraufwand }\end{array}$ \\
\hline & Größe & $\begin{array}{l}\text { Lage der } \\
\text { ZBE }\end{array}$ & mittel & kaum & $\begin{array}{l}\text { Transportweg, -länge, -geometrie } \\
\text { Vormontageflächen }\end{array}$ & $\begin{array}{l}\text { Zugänglichkeit i.w.s. } \\
\text { Notwendige Ausrüstung }\end{array}$ \\
\hline & Gliederung & $\begin{array}{l}\text { horizontal } \\
\text { vertikal }\end{array}$ & groß & kaum & $\begin{array}{l}\text { Hebezeugeinsatz } \\
\text { Zugänglichkeit für ME, AM } \\
\text { Möglichkeit der Blockmontage }\end{array}$ & $\begin{array}{l}\text { Flächenbedarf für Auf-, Um und } \\
\text { Abrüsten }\end{array}$ \\
\hline & $\begin{array}{l}\text { Gliederung } \\
\text { in } \\
\text { Teilanlagen }\end{array}$ & $\begin{array}{l}\text { funktions- } \\
\text { fähig }\end{array}$ & groß & kaum & $\begin{array}{l}\text { Hebezeugeinsatz } \\
\text { Zugänglichkeit für Montageeinheit, AM } \\
\text { Verflechtungsdichte }\end{array}$ & In Verbindung mit Montageorganisation \\
\hline & Bauwelse & $\begin{array}{l}\text { Freibau } \\
\text { Teilfreibau } \\
\text { Umhausung }\end{array}$ & sehr groß & kaum & $\begin{array}{l}\text { Hebezeugeinsatz } \\
\text { Zugänglichkeit für Montageeinheit, AM } \\
\text { Kombinierte Bau-Ausrüstungs-Montage }\end{array}$ & $\begin{array}{l}\text { Verfahren bestimmt Bauweise } \\
\text { Wechselwirkung Bau - Ausrüstung }\end{array}$ \\
\hline & \multirow[t]{2}{*}{$\begin{array}{l}\text { Ausrüstungs- } \\
\text { auswahl }\end{array}$} & $\begin{array}{l}\text { verfahrens- } \\
\text { technisch } \\
\text { abhängig } \\
\text { dimensioniert }\end{array}$ & groß & kaum & $\begin{array}{l}\text { Masse/Geometrie/Abmessung } \\
\text { Anzahl, Art und Lage der Fügeflächen }\end{array}$ & \multirow[t]{2}{*}{$\begin{array}{l}\text { S. Ersatzkriterien } \\
\text { Montageeinheit }\end{array}$} \\
\hline & & $\begin{array}{l}\text { verfahrens- } \\
\text { technisch } \\
\text { unabhängig } \\
\text { dimensioniert }\end{array}$ & groB & kaum & $\begin{array}{l}\text { Zugänglichkeit zum Einbauort } \\
\text { Hebezeugeinsatz } \\
\text { Transportlage - Einbaulage } \\
\text { Justieraufwand }\end{array}$ & \\
\hline & \multirow[t]{2}{*}{$\begin{array}{l}\text { Ausrüstungs- } \\
\text { anordnung }\end{array}$} & $\begin{array}{l}\text { verfahrens- } \\
\text { bedingt }\end{array}$ & groß & kaum & $\begin{array}{l}\text { Zugänglichkeit für ME, AM } \\
\text { Blockmontagemöglichkeiten }\end{array}$ & \multirow[t]{2}{*}{ Wechselwirkung Instandhaltung } \\
\hline & & $\begin{array}{l}\text { nicht verfah- } \\
\text { rens bedingt }\end{array}$ & groß & kaum & $\begin{array}{l}\text { Zugänglichkeit für ME, AM } \\
\text { Einsatz moderner Montageverfahren }\end{array}$ & \\
\hline & Kopplungen & $\begin{array}{l}\text { Verlegungs- } \\
\text { art }\end{array}$ & groß & kaum & $\begin{array}{l}\text { Vorfertigungsgrad } \\
\text { Prüf- und Kontrollmöglichkeiten }\end{array}$ & \\
\hline
\end{tabular}

\begin{tabular}{|c|c|c|c|}
\hline $\begin{array}{l}\text { Charakteristisches } \\
\text { Hauptmerkmal }\end{array}$ & $\begin{array}{l}\text { Zusatz- } \\
\text { Merkmal }\end{array}$ & Beurteilungskriterien & Bemerkungen \\
\hline Masse & Schwertpunktlage & $\begin{array}{l}\text { Notwendige Hebezeuge } \\
\text { Montagehilfsmittel (spezielle) } \\
\text { Vormontagegrad }\end{array}$ & $\begin{array}{l}\text { Beschaffenheit der } \\
\text { Transportwege } \\
\text { (Erdstabilisierungsmaßnahmen) }\end{array}$ \\
\hline Abmessungen & Schwertpunktlage & $\begin{array}{l}\text { Zugänglichkeit zum Einbauort } \\
\text { Hebezeugeinsatz } \\
\text { Montagehilfsmittel (spezielle) } \\
\text { Vormontagegrad }\end{array}$ & \\
\hline Geometrie & $\begin{array}{l}\text { Stabilität } \\
\text { (Lastfall Montage) }\end{array}$ & $\begin{array}{l}\text { Transportfähigkeit (Zuführen) } \\
\text { Montagehilfsmittel (spezielle) } \\
\text { Anschlagmöglichkeiten } \\
\text { Montagestabilisierung } \\
\text { Komplettierung (Blockbildung) }\end{array}$ & $\begin{array}{l}\text { Mehraufwand für zeitweilige } \\
\text { Stabilisierung }\end{array}$ \\
\hline $\begin{array}{l}\text { Werkstoff; Anzahl, } \\
\text { Lage und Art der } \\
\text { Fügeflächen }\end{array}$ & Transport -Einbaulage & $\begin{array}{l}\text { Zugänglichkeit für Arbeitsmittel, Arbeitskräfte } \\
\text { Aufwand für Zuführen } \\
\text { Aufwand für Positionieren } \\
\text { Aufwand für Lagesichern } \\
\text { Aufwand für Fügen } \\
\text { Rüstaufwand, Blockbildung }\end{array}$ & Montagestabilisierung \\
\hline Komplettierung & $\begin{array}{l}\text { Vormontage } \\
\text { Schwerpunktlage }\end{array}$ & $\begin{array}{l}\text { Hebezeugeinsatz } \\
\text { Zugänglichkeit für Anschlagmöglichkeiten } \\
\text { Montagehilfsmittel (spezielle) } \\
\text { Blockbildung, Zuführbarkeit } \\
\text { Aufwand für Vor- und Nachbereitung } \\
\text { Rüstaufwand, Umsetzen des Hebezeuges }\end{array}$ & \\
\hline Einbausituation & Transport - Einbaulage & $\begin{array}{l}\text { Zugänglichkeit zum Einbauort } \\
\text { Aufwand für Positionieren } \\
\text { zusätzliche Anschlagmöglichkeiten } \\
\text { Rüstaufwand } \\
\text { zusätzliche Montagehilfsmittel }\end{array}$ & \\
\hline Funktionsfähigkeit & Druck-, Dichtheitsprüfung & $\begin{array}{l}\text { Aufwand für Prüf- und Kontrollmaßnahmen } \\
\text { Rüstaufwand }\end{array}$ & \\
\hline
\end{tabular}

